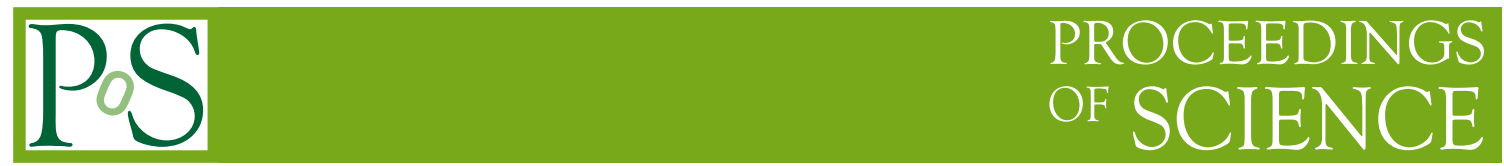

\title{
Weak Bosons and Jets at the LHC
}

\section{Tom Melia* ${ }^{* \dagger}$}

University of Oxford Theoretical Physics

E-mail: t.melial@physics.ox.ac.uk

In this talk, I outline theoretical predictions for weak boson pair production in association with two jets at the LHC. I will discuss the next-to-leading order QCD corrections to the processes $p p \rightarrow W^{+} W^{+} j j$ and $p p \rightarrow W^{+} W^{-} j j$, and the interfacing of $p p \rightarrow W^{+} W^{+} j j$ with a parton shower using the POWHEG BOX framework.

10th International Symposium on Radiative Corrections (Applications of Quantum Field Theory to Phenomenology) - Radcor2011

September 26-30, 2011

Mamallapuram, India

\footnotetext{
*Speaker.

${ }^{\dagger}$ Based on work done in collaboration with Kirill Melnikov, Paolo Nason, Raoul Röntsch, and Giulia Zanderighi see Refs. [1, 2, 3].
} 

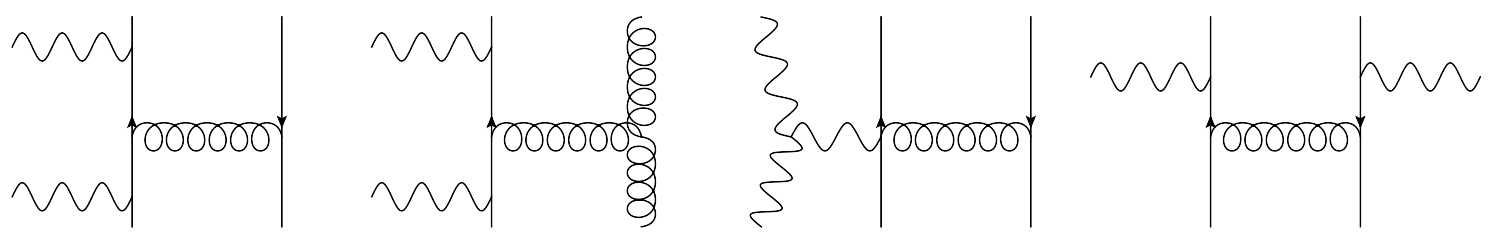

Figure 1: Types of Feynman graphs encountered in a tree-level calculation of weak boson pair production in association with two jets. Wavy lines depict weak bosons and spiralled lines are gluons. Quark flavour labels and weak boson labels $\left(W^{+} / W^{-} / Z^{0}\right)$ have been deliberately suppressed to highlight the general topology of the graphs which may contribute to different processes.

\section{Introduction and Motivation}

Precision calculations of standard model processes are essential for interpreting the signals measured at the Large Hadron Collider (LHC) and for fully realising the discovery potential of this experiment. Next-to-leading order (NLO) calculations in perturbative QCD have proved very successful when used in Tevatron analyses, and are a good way to reduce theoretical uncertainties in a description of a given process. On top of this, merging an NLO calculation with a parton shower provides a realistic hadron-level prediction for an event whilst maintaining NLO accuracy for inclusive observables.

In this talk I shall discuss the production of a pair of weak bosons in association with jets specifically the two processes $p p \rightarrow W^{+} W^{+} j j$ and $p p \rightarrow W^{+} W^{-} j j$. I will describe the computation of the NLO QCD corrections to both processes, as well as the merging of $p p \rightarrow W^{+} W^{+} j j$ with a parton shower, done in the framework of the POWHEG BOX [4].

Figure 1 depicts the structure of some of the types of Feynman graphs one encounters in a tree-level calculation of weak boson pair production along with two jets. All of these types of graph contribute towards the process $p p \rightarrow W^{+} W^{-} j j$, but only graphs of the type shown in the far right of the figure contribute towards $p p \rightarrow W^{+} W^{+} j j$. Here, charge conservation requires the two $W^{+}$bosons to be emitted from separate quark lines and this leads to an unusual theoretical property - the cross section for this process remains finite even if the requirement that two jets are observed is lifted. This will be investigated later on, and I will present results for $W^{+} W^{+}+n$ jets, where $n=0,1,2$. The calculation of $p p \rightarrow W^{+} W^{+} j j$ can be seen as a stepping stone to the calculation of $p p \rightarrow W^{+} W^{-} j j$, since it involves a small subset of the Feynman graphs needed for the latter.

Both processes are $2 \rightarrow 4$ processes, and to calculate the QCD corrections to them one needs to deal with one-loop, six-point tensor integrals of relatively high rank. There is thus a theoretical incentive in performing these calculations and much progress has been made over the past few years in the methods used to compute them - this will be discussed in the following section. But before this, I will go on to discuss the study of both processes at the LHC in a bit more detail.

\section{$1.1 W^{+} W^{+} j j$ at the LHC}

At $\sqrt{s}=14 \mathrm{TeV}$, the cross-section for this process is about $1 \mathrm{pb}\left(40 \%\right.$ of this for $\left.W^{-} W^{-} j j\right)$ and is therefore accessible. In the following we take the $W^{+}$bosons to both decay leptonically, giving rise to a nearly background-free signature which involves same-sign leptons. This is an interesting 
process to study in its own right, but there are other reasons to study it: $p p \rightarrow W^{+} W^{+} j$ is a background to physics both within and beyond the standard model. For example, it is possible to use same-sign lepton pairs to study double parton scattering at the LHC [5], to which $p p \rightarrow W^{+} W^{+} j j$ is a background. Beyond the standard model, resonant slepton production in R-parity violating SUSY models [6], diquark production [7], and doubly charged Higgs boson production [8] are examples of processes which also lead to a signature of two same-sign leptons, missing energy, and jets.

\section{$1.2 W^{+} W^{-} j j$ at the $\mathrm{LHC}$}

The production of a $W^{+} W^{-}$boson pair in association with zero, one or two jets is an important background to Higgs boson production, especially when the decay $H \rightarrow W^{+} W^{-}$opens up. Although most of the sensitivity in Higgs boson searches comes from the zero jet processes, which have the largest cross-section, the production of a Higgs boson in association with two jets is also relevant - about $10 \%$ of Higgs events at the LHC involve two jets $[9,10]$. The production of a Higgs boson via weak boson fusion (WBF) also has a sizeable cross-section. The signature of this process includes two forward tagging jets and $p p \rightarrow W^{+} W^{-} j j$ is an irreducible background to this. As we did for $W^{+} W^{+} j j$, in the following we will take both $W$ bosons to decay leptonically. The resulting signature of two opposite-sign leptons, jets and missing energy is also a background to a classic beyond the standard model physics search.

\section{Method of calculation}

\subsection{The NLO QCD corrections}

NLO QCD calculations of processes involving more than five particles is difficult. For the virtual amplitude, the number of Feynman diagrams needing evaluation grows factorially with the number of particles in the process. In addition to this, the one-loop tensor integrals which need to be computed become more involved. However, a refinement of traditional computation methods, as well as the development of new techniques based on unitarity and on-shell methods, have seen a significant growth in the number of $2 \rightarrow 4$ processes (and even a $2 \rightarrow 5$ process) known at NLO in the past few years (see [11] for a recent review). Platforms for the automation of NLO-accurate processes are currently being developed (see e.g. [12, 13, 14, 15]).

As described in detail in the papers [1,3], the technique of $D$-dimensional generalised unitarity [16] was used to obtain the virtual part of the amplitude for the QCD processes $p p \rightarrow W^{+} W^{+} j j$ and $p p \rightarrow W^{+} W^{-} j j$. It is worth pointing out that, as currently formulated, on-shell methods require working with an ordering of external lines - these are colour ordered or primitive amplitudes. It is only colour-charged particles which are ordered in primitive amplitudes and so all possible insertions of the colourless weak bosons must be considered for any tree-level or one-loop primitive amplitude. The $D$-dimensional unitarity cuts reduce one-loop primitive amplitudes to products of tree-level helicity amplitudes, and a certain amount of difficulty exists in ensuring no over-counting takes place when combining the cuts of different parent diagrams. Nevertheless, this is just bookkeeping and these two calculations demonstrated that unitarity methods can deal with more complicated, colourless final states. The tree-level helicity amplitudes themselves are calculated using Berend-Giele recursion relations [17]. 

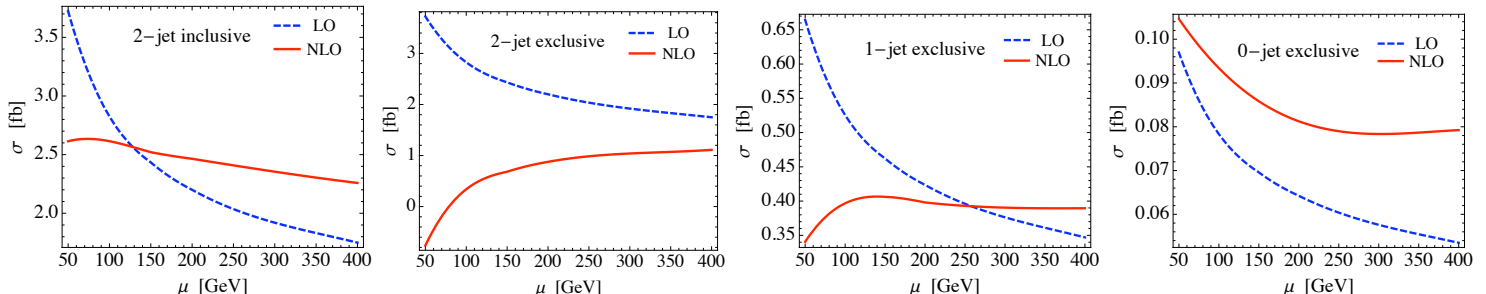

Figure 2: The dependence on factorisation and renormalisation scales of cross-sections for $p p \rightarrow e^{+} \mu^{+} v_{e} v_{\mu}+n$ jets, $n=0,1,2$ at leading and next-to-leading order in perturbative QCD. Here $\mu_{\mathrm{F}}=\mu_{\mathrm{R}}=\mu$.

\subsection{Merging with a parton shower}

Methods which include both the benefits of an NLO calculation and a parton shower model (NLO+PS generators) have become available in recent years - two frameworks are currently being used for collider physics: MC@NLO [18] and POWHEG [19]. A general computer framework for building a POWHEG implementation of an arbitrary NLO process exists - the POWHEG BOX [4]. Here, one needs only to supply a few ingredients: phase-space and flavour information, the Born and real matrix elements and the virtual matrix elements for a given NLO process. The implementation of $p p \rightarrow W^{+} W^{+} j j$ in the POWHEG BOX is reported in [2]. This was the first time a $2 \rightarrow 4$ process was implemented in a NLO+PS generator.

Since all of the ingredients needed by the POWHEG BOX were already known from [1], the POWHEG implementation of this process did not present any special problem, except for a nontrivial issue of high computational demands coming from the virtual corrections. The technical details of how this problem was dealt with are described in detail in [2]. The resulting code is public and is available at the website [20].

\section{Results}

\subsection{Selected results for $p p \rightarrow W^{+} W^{+} j j$ at the LHC}

First I will present results from the NLO calculation of $p p \rightarrow W^{+} W^{+} j j$, taken from [1]. We consider proton-proton collisions at a center-of-mass energy $\sqrt{s}=14 \mathrm{TeV}$. We require leptonic decays of the $W$-bosons and consider the final state $e^{+} \mu^{+} v_{e} v_{\mu}$. The $W$-bosons are on the massshell and we neglect quark flavour mixing. We impose standard cuts on lepton transverse momenta $p_{\perp, l}>20 \mathrm{GeV}$, missing transverse momentum $p_{\perp, \text { miss }}>30 \mathrm{GeV}$ and charged lepton rapidity $\left|\eta_{l}\right|<$ 2.4. We define jets using anti- $k_{\perp}$ algorithm, with $R=0.4$ and with a transverse momentum cut $p_{\perp, j}=30 \mathrm{GeV}$ on the two jets. The mass of the $W$-boson is taken to be $m_{W}=80.419 \mathrm{GeV}$, the width $\Gamma_{W}=2.140 \mathrm{GeV} . W$ couplings to fermions are obtained from $\alpha_{\mathrm{QED}}\left(m_{Z}\right)=1 / 128.802$ and $\sin ^{2} \theta_{W}=0.2222$. We use MSTW08LO parton distribution functions for leading order and MSTW08NLO for next-to-leading order computations, corresponding to $\alpha_{s}\left(M_{Z}\right)=0.13939$ and $\alpha_{s}\left(M_{Z}\right)=0.12018$ respectively. We do not impose lepton isolation cuts. All results discussed below apply to the QCD production $p p \rightarrow W^{+} W^{+} j j$; the electroweak contribution to this process is ignored. 


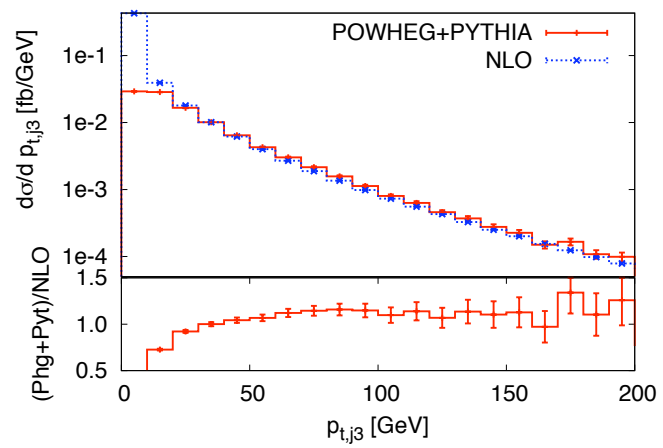

Figure 3: The kinematic distribution for the transverse momentum of the third hardest jet in the QCD production of $p p \rightarrow e^{+} \mu^{+} v_{e} v_{\mu}+2$ jets. The pure NLO result and the result with POWHEG+PYTHIA are both shown.

Figure 2 shows the dependence of the production cross-sections for $p p \rightarrow e^{+} \mu^{+} v_{e} v_{\mu}+n$ jets on the renormalisation and factorisation scales, which we set equal to each other. Considering the range of scales $50 \mathrm{GeV} \leq \mu \leq 400 \mathrm{GeV}$, we find the two-jet inclusive cross-section to be $\sigma^{\mathrm{LO}}=2.7 \pm 1.0 \mathrm{fb}$ at leading order and $\sigma^{\mathrm{NLO}}=2.44 \pm 0.18 \mathrm{fb}$ at next-to-leading order. The forty percent scale uncertainty at leading order is reduced to less than ten percent at NLO. We observe similar stabilization of the scale dependence for the 0 - and 1 -jet exclusive multiplicities. Combining these cross-sections we obtain a total NLO cross-section of about $2.90 \mathrm{fb}$ for $p p \rightarrow e^{+} \mu^{+} v_{e} v_{\mu}$ inclusive production. This implies about $60 e^{+} \mu^{+}+e^{+} e^{+}+\mu^{+} \mu^{+}$events per year at the LHC with $10 \mathrm{fb}^{-1}$ annual luminosity. While this is not a gigantic number, such events will have a very distinct signature, so they will definitely be seen and it will be possible to study them.

The dramatic change in the two-jet exclusive cross-section apparent from figure 2 is discussed and investigated in [1]. We find that the feature observed here, that the two-jet exclusive is significantly smaller than the two-jet inclusive, remains present when we increase the jet cut and so allow for greater perturbative convergence of the exclusive cross section. This smallness implies that quite a large fraction of events in $p p \rightarrow e^{+} \mu^{+} v_{e} v_{\mu}+\geq 2$ jets have a relatively hard third jet. This feature may be useful for rejecting contributions of $p p \rightarrow W^{+} W^{+} j j$ when looking for multiple parton scattering.

Next I present results from the POWHEG implementation of $p p \rightarrow W^{+} W^{+} j j$, taken from the paper [2]. Here the set-up is as described above, but we consider $p p$ collisions at a different centre of mass energy: $\sqrt{s}=7 \mathrm{TeV}$. A dynamic scale is used for the renormalisation and factorisation scales:

$$
\mu_{R}=\mu_{F}=\left(p_{\perp, 1}+p_{\perp, 2}+E_{\perp, W_{1}}+E_{\perp, W_{2}}\right) / 2, \quad E_{\perp, W}=\sqrt{m_{W}^{2}+p_{\perp, W}^{2}},
$$

where $p_{\perp, W_{1}}, p_{\perp, W_{2}}, p_{\perp, 1}$ and $p_{\perp, 2}$ are the transverse momenta of the two $W \mathrm{~s}$ and the two emitted partons in the underlying Born configuration.

With no jet cuts, but with the leptonic cuts described above, we find the cross-section for to be $1.11 \pm 0.01 \mathrm{fb}$ for the pure NLO result, and a slightly lower cross-section of $1.06 \pm 0.01 \mathrm{fb}$ when events are generated by POWHEG and are subsequently showered with PYTHIA. A comparison 


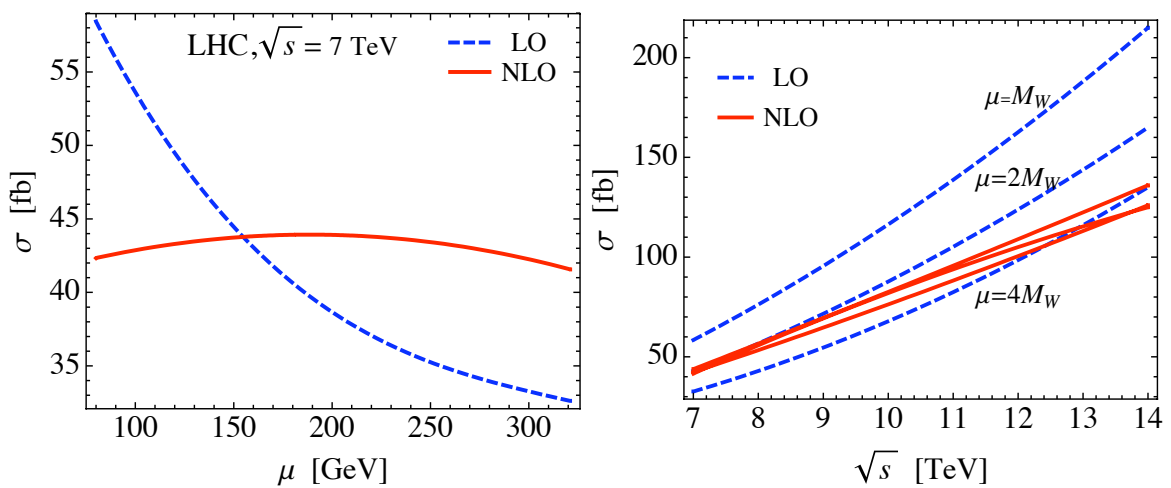

Figure 4: Left pane: the production cross-section of the process $p p \rightarrow\left(W^{+} \rightarrow v_{e} e^{+}\right)\left(W^{-} \rightarrow \mu^{-} \bar{v}_{\mu}\right) j j$ at the $7 \mathrm{TeV}$ LHC in dependence on the factorisation and renormalisation scales $\mu_{F}=\mu_{R}=\mu$ at both LO and NLO in perturbative QCD. Right pane: the dependence of the cross-section on centre of mass energy $\sqrt{s}$ with LO results in dashed blue and NLO results in solid red. Three choices of $\mu$ are shown: $\mu=m_{W}, 2 m_{w}, 4 m_{W}$.

of kinematic distributions was carried out in [2] and for the most part, there was good agreement between the NLO and the POWHEG+PYTHIA results. However, there were some distributions which showed expected and marked changes, one of which I shall highlight in this talk. Figure 3 shows the transverse momentum of the third-hardest jet. Since at NLO it is only the real radiation which contributes to this distribution, we see a divergence for small $p_{\perp, j_{3}}$ in the pure NLO result. In contrast one can see the Sudakov peak in the POWHEG + PYTHIA result, and the distribution goes to zero as $p_{\perp, j_{3}} \rightarrow 0$.

\subsection{Selected results for $p p \rightarrow W^{+} W^{-} j j$ at the LHC}

Here I will present selected results from the calculation of $p p \rightarrow W^{+} W^{-} j j$, taken from the paper [3]. Here the $W$ bosons decay leptonically: $W^{+} W^{-} j j \rightarrow e^{+} \mu^{-} v_{e} \bar{v}_{\mu} j j$. The full results with generic opposite-sign leptons can be obtained from these by multiplying by a factor of four. We use the same leptonic cuts and electroweak input parameters as were described in the $p p \rightarrow W^{+} W^{+} j j$ results section above. However, here of course a jet cut must be applied and two jets observed in order to obtain a finite cross-section: we take $p_{\perp, j}>30 \mathrm{GeV}$ and $\left|\eta_{j}\right|<3.2$.

Figure 4 shows the dependence of the production cross-section on renormalisation and factorisation scales, which are again set equal to each other, at a centre of mass energy $\sqrt{s}=7 \mathrm{TeV}$. The dependence of the cross-section on centre of mass energy is also shown in figure 4. One observes a dramatic reduction in scale dependence in going from leading order to next-to-leading order. Considering a range of scales $m_{W}<\mu<4 m_{W}$ we obtain a cross section at leading order $\sigma_{L O}=46 \pm 13 \mathrm{fb}$ and at NLO $\sigma_{N L O}=42 \pm 1 \mathrm{fb}$. Assuming fifty percent efficiency, with $5 \mathrm{fb}^{-1}$ of data at the $7 \mathrm{TeV}$ run of the LHC, we expect about 400 dilepton events $e^{+} \mu^{-}, e^{+} e^{-}, \mu^{+} e^{-}, \mu^{+} \mu^{-}$. It is interesting that at NLO, the dependence of the cross-section on centre of mass energy $\sqrt{s}$ is almost linear. If one defines an 'optimal' scale choice to be the choice of scale for which NLO corrections are smallest then this 'optimal' scale shifts from $2 m_{W}$ at $7 \mathrm{TeV}$ to $4 m_{W}$ at $14 \mathrm{TeV}$.

Finally I present two kinematic distributions for this process which are relevant for a Higgs boson search at the LHC. The left pane of figure 5 plots the relative azimuthal angle between the 

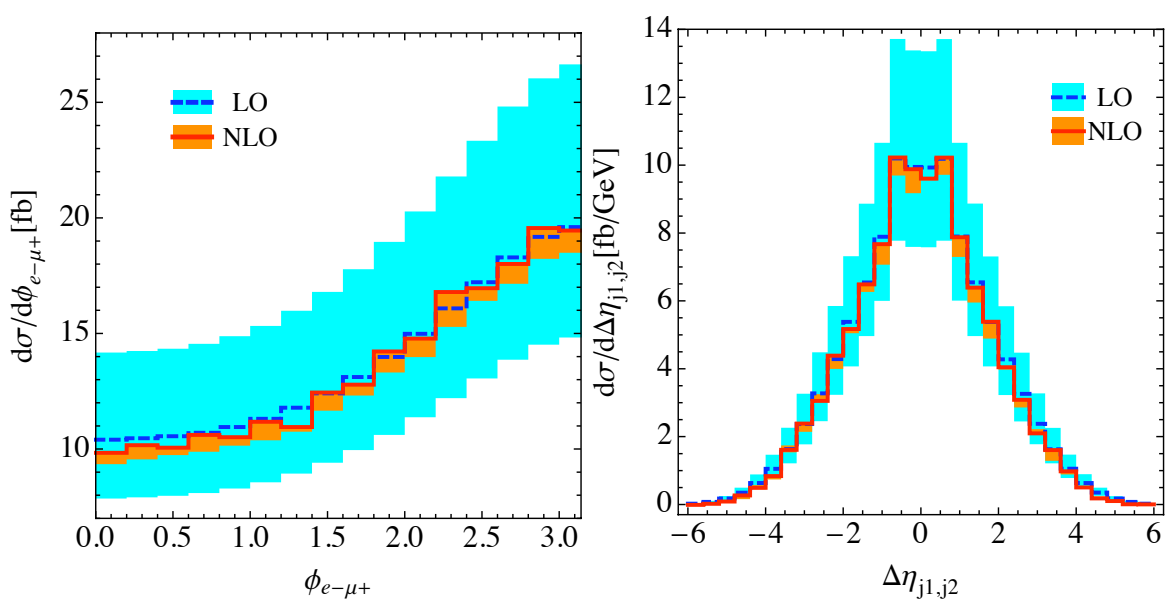

Figure 5: Distributions of lepton opening angle and jet pseudorapidity difference for the process $p p \rightarrow\left(W^{+} \rightarrow v_{e} e^{+}\right)\left(W^{-} \rightarrow \mu^{-} \bar{v}_{\mu}\right) j j$ at the $7 \mathrm{TeV}$ LHC. LO results are shown in blue, NLO results in red. The uncertainty bands are for scale $m_{W}<\mu<4 m_{W}$ and the solid lines show the results at $\mu=2 m_{W}$.

leptons which peaks at $\phi_{e^{-}} \mu^{+}=\pi$. This is in contrast to leptons produced via the mechanism $H \rightarrow W W \rightarrow e^{+} \mu^{-} v v$ where this angle tends to be small. The pseudorapidity difference between the two leading jets, $\Delta \eta_{j 1 j 2}=\eta_{j 1}-\eta_{j 2}$, is plotted in the right pane of figure 5 . This is a useful distribution for studying Higgs boson production via WBF - this mechanism leads to jets which tend to have a large $\left|\Delta \eta_{j 1 j 2}\right|$. For a Higgs produced via gluon fusion and, as we see here for $p p \rightarrow W^{+} W^{-} j j$, this distribution is peaked around $\left|\Delta \eta_{j 1 j 2}\right|=0$. The significant reduction in theoretical scale uncertainties can also be seen in these distributions, and there is no observed shape change in going from LO to NLO. These observations were typical of all kinematic distributions considered in [3].

\section{Conclusion}

In this talk I have presented the NLO QCD corrections for the process $p p \rightarrow W^{+} W^{+} j j$ and the process $p p \rightarrow W^{+} W^{-} j j$ which were computed using the method of $D$-dimensional generalised unitarity. A significant reduction in the theoretical uncertainties of an LHC prediction is observed for both processes. The process $p p \rightarrow W^{+} W^{+} j j$ has been implemented in the POWHEG BOX which matches the NLO result with a parton shower. I look forward to measurements of pairs of weak bosons and jets at the LHC.

\section{Acknowledgements}

I wish to thank the organisers of RADCOR2011 for a really fantastic conference and for providing financial support. This talk is based on work done in collaboration with Kirill Melnikov, Paolo Nason, Raoul Röntsch, and Giulia Zanderighi and draws on the papers [1, 2, 3]. This research is supported by the British Science and Technology Facilities Council. 


\section{References}

[1] T. Melia, K. Melnikov, R. Rontsch and G. Zanderighi, "Next-to-leading order QCD predictions for $W^{+} W^{+} j j$ production at the LHC," JHEP 1012, 053 (2010).

[2] T. Melia, P. Nason, R. Rontsch and G. Zanderighi, " $W^{+} W^{+}$plus dijet production in the POWHEG BOX,” Eur. Phys. J. C 71, 1670 (2011).

[3] T. Melia, K. Melnikov, R. Rontsch and G. Zanderighi, "NLO QCD corrections for $W^{+} W^{-}$pair production in association with two jets at hadron colliders," Phys. Rev. D 83, 114043 (2011).

[4] S. Alioli, P. Nason, C. Oleari and E. Re, "A general framework for implementing NLO calculations in shower Monte Carlo programs: the POWHEG BOX,” JHEP 1006 (2010) 043 [arXiv:1002.2581 [hep-ph]].

[5] J. R. Gaunt, C. -H. Kom, A. Kulesza and W. J. Stirling, "Same-sign W pair production as a probe of double parton scattering at the LHC," Eur. Phys. J. C 69 (2010) 53 [arXiv:1003.3953 [hep-ph]].

[6] See e.g. H. K. Dreiner, S. Grab, M. Kramer and M. K. Trenkel, "Supersymmetric NLO QCD corrections to resonant slepton production and signals at the Tevatron and the CERN LHC," Phys. Rev. D 75 (2007) 035003 [hep-ph/0611195].

[7] See e.g. T. Han, I. Lewis and T. McElmurry, "QCD Corrections to Scalar Diquark Production at Hadron Colliders,” JHEP 1001 (2010) 123 [arXiv:0909.2666 [hep-ph]].

[8] See e.g. J. Maalampi and N. Romanenko, "Single production of doubly charged Higgs bosons at hadron colliders," Phys. Lett. B 532 (2002) 202 [hep-ph/0201196].

[9] C. Anastasiou, G. Dissertori, M. Grazzini, F. Stockli and B. R. Webber, "Perturbative QCD effects and the search for a H $\rightarrow$ WW $\rightarrow$ l nu 1 nu signal at the Tevatron," JHEP 0908 (2009) 099 [arXiv:0905.3529 [hep-ph]].

[10] J. M. Campbell, R. K. Ellis and G. Zanderighi, "Next-to-Leading order Higgs +2 jet production via gluon fusion,” JHEP 0610 (2006) 028 [hep-ph/0608194].

[11] R. K. Ellis, Z. Kunszt, K. Melnikov and G. Zanderighi, “One-loop calculations in quantum field theory: from Feynman diagrams to unitarity cuts," arXiv:1105.4319 [hep-ph].

[12] G. Cullen et al., “Automated One-Loop Calculations with GoSam,” arXiv:1111.2034 [hep-ph].

[13] G. Bevilacqua et al., "HELAC-NLO,” arXiv:1110.1499 [hep-ph].

[14] V. Hirschi, R. Frederix, S. Frixione, M. V. Garzelli, F. Maltoni and R. Pittau, "Automation of one-loop QCD corrections," JHEP 1105 (2011) 044 [arXiv:1103.0621 [hep-ph]].

[15] C. F. Berger et al., "An Automated Implementation of On-Shell Methods for One-Loop Amplitudes," Phys. Rev. D 78 (2008) 036003 [arXiv:0803.4180 [hep-ph]].

[16] W. T. Giele, Z. Kunszt and K. Melnikov, "Full one-loop amplitudes from tree amplitudes," JHEP 0804, 049 (2008).

[17] F. A. Berends and W. T. Giele, "Recursive Calculations for Processes with n Gluons," Nucl. Phys. B 306 (1988) 759.

[18] S. Frixione and B. R. Webber, "Matching NLO QCD computations and parton shower simulations," JHEP 0206 (2002) 029 [arXiv:hep-ph/0204244].

[19] P. Nason, “A New method for combining NLO QCD with shower Monte Carlo algorithms," JHEP 0411 (2004) 040 [hep-ph/0409146].

[20] http://powhegbox.mib.infn.it/ 